Article

\title{
Curcumin Nanoparticles and Their Cytotoxicity in Docetaxel-Resistant Castration-Resistant Prostate Cancer Cells
}

\author{
Irin Tanaudommongkon ${ }^{1}$, Asama Tanaudommongkon ${ }^{1}$, Priyanka Prathipati ${ }^{1}$, \\ Joey Trieu Nguyen ${ }^{1}$, Evan T. Keller ${ }^{2}$ D and Xiaowei Dong ${ }^{1, *}$ \\ 1 Department of Pharmaceutical Sciences, University of North Texas Health Science Center, Fort Worth, \\ TX 76107, USA; it0046@live.unthsc.edu (I.T.); at0371@live.unthsc.edu (A.T.); \\ priyankaprathipati@gmail.com (P.P.); jhn0043@my.unthsc.edu (J.T.N.) \\ 2 Department of Urology and Biointerfaces Institute, University of Michigan, Ann Arbor, MI 48109, USA; \\ etkeller@umich.edu \\ * Correspondence: xiaowei.dong@unthsc.edu; Tel.: +1-817-735-2785
}

Received: 7 July 2020; Accepted: 23 July 2020; Published: 30 July 2020

\begin{abstract}
Most prostate cancer patients develop resistance to anti-androgen therapy. This is referred to as castration-resistant prostate cancer (CRPC). Docetaxel (DTX) is the mainstay treatment against CRPC. However, over time patients eventually develop DTX resistance, which is the cause of the cancer-related mortality. Curcumin (CUR) as a natural compound has been shown to have very broad pharmacological activities, e.g., anti-inflammatory and antioxidant properties. However, CUR is very hydrophobic. The objective of this study was to develop CUR nanoparticles (NPs) and evaluate their cytotoxicity in DTX-resistant CRPC cells for the treatment of DTX-resistant CRPC. We tested solubility of CUR in different lipids and surfactants. Finally, Miglyol 812 and D-alpha-tocopheryl poly (ethylene glycol) succinate 1000 (TPGS) were chosen to prepare lipid-based NPs for CUR. We fully characterized CUR NPs that had particle size $<150 \mathrm{~nm}$, high drug loading $(7.5 \%)$, and entrapment efficiency (90\%). Moreover, the CUR NPs were successfully lyophilized without using cryoprotectants. We tested the cytotoxicity of blank NPs, free CUR, and CUR NPs in sensitive DU145 and PC3 cells as well as their matching docetaxel-resistant cells. Cytotoxicity studies showed that blank NPs were very safe for all tested prostate cancer cell lines. Free CUR overcame the resistance in PC 3 cells, but not in DU145 cells. In contrast, CUR NPs significantly increased CUR potency in all tested cell lines. Importantly, CUR NPs completely restored CUR potency in both resistant DU145 and PC3 cells. These results demonstrate that the CUR NPs have potential to overcome DTX resistance in CRPC.
\end{abstract}

Keywords: sensitive and resistant prostate cancer cells; cytotoxicity; therapeutic agents; lipid-based nanoparticles; P-glycoprotein

\section{Introduction}

Prostate cancer (PCa) is the second-most-frequent cancer diagnosis in men and the fifth leading cause of cancer death worldwide [1]. Anti-androgen is the first line treatment for PCa. However, most patients will develop resistance to anti-androgen therapy and high metastases, which is referred to as castration-resistant prostate cancer (CRPC) [2]. Docetaxel (DTX) was approved by the Food and Drug Administration (FDA) as the mainstay treatment against CRPC and has been shown to improve the survival rate of men with metastatic CRPC [3]. However, over time patients eventually develop DTX resistance, which is the cause of the cancer-related mortality. Multiple mechanisms exist for drug resistance and a drug is not confronted by a single mechanism. One well-known mechanism of drug resistance is overexpression of P-glycoprotein (P-gp), a transporter that can efflux the drugs out of 
cells [4]. Fairly compelling data showed the correlation between P-gp overexpression and poor clinical outcomes in many cancers, including PCa [5,6]. Indeed, overexpression of P-gp is the main cause of resistance in docetaxel-resistant DU145 cells [7]. To date, the use of P-gp inhibitors to enhance efficacy in drug-resistant cancers has not been successful in clinical trials [8]. Because of the lack of specificity, P-gp inhibitors also inhibit P-gp in normal cells, leading to significant side effects. Even though new cytotoxicity agents, e.g., cabazitaxel, were developed to avoid P-gp recognition, tumor heterogeneity still generates drug resistance such as up-regulation of apoptosis genes and microtubulin alterations in CRPC [7]. Thus, a new therapeutic agent is needed to overcome multiple resistance mechanisms in CRPC.

Curcumin (CUR) is a bioactive compound extracted from the rhizome of the Curcuma longa plant. It has a low molecular weight and is a yellow, lipophilic polyphenolic compound. Due to a wide range of biological and pharmacological activities, CUR contains anti-inflammatory and antioxidant properties, and has been studied as a chemopreventative and chemotherapeutic agent [9]. CUR is also involved in cell cycle control and stimulation of apoptosis. It modulates autophagy and has inhibitory effects on tumor angiogenesis and metastasis in numerous human cancers [10,11]. Combination of taxanes and CUR has demonstrated synergistic cytotoxicity in sensitive PC3 cells [12]. However, the influence of CUR for DTX-resistant CRPC is unknown.

The application of CUR in clinical trials has been limited due to hydrophobicity, low water solubility, instability, and poor pharmacokinetics in spite of its superior properties [13-15]. Nanoparticles (NPs) were designed to deliver CUR in order to enhance its efficacy in cancers. NPs have the potential to protect drugs from degradation, enhance drug stability, control the drug release, alter and improve the pharmacokinetic and pharmacodynamic properties, and decrease toxicity [16,17]. Lipid-based NPs were chosen due to the advantages of being able to pass through leaky and hyperpermeable tumor vasculature and accumulate in the tumor vicinity by utilizing the enhanced permeability and retention (EPR) effect [18]. Importantly, they have been explored to overcome drug resistance in cancers. Some surfactants have been shown the potential to inhibit P-gp function. For example, D-alpha-tocopheryl poly (ethylene glycol) succinate 1000 (TPGS) is a vitamin E derivative and has been approved by the FDA as a safe pharmaceutical excipient used in many formulations. TPGS inhibits drug efflux through an allosteric modulation of P-gp [19].

The aim of this study was to develop CUR NPs and evaluate their cytotoxicity in DTX-resistant CRPC cells for the treatment of DTX-resistant CRPC. We used Miglyol 812 and TPGS to prepare NPs that encapsulated CUR, so called CUR MT NPs. We fully characterized the NPs in terms of particle size, zeta potential, polydispersity index (P.I.), drug entrapment efficiency (EE), drug loading (DL), differential scanning calorimetry (DSC) analysis, stability studies, and in vitro release study. Moreover, we tested the cytotoxicity of blank MT NPs, free CUR and CUR MT NPs in sensitive DU145 and PC3 cells and their matching DTX-resistant cells.

\section{Materials and Methods}

\subsection{Chemicals and Reagents}

CUR was purchased from Adipogen Corporation (San Diego, CA, USA). Phosphate buffer saline was purchased from American Type Culture Collection (ATCC) (Manassas, VA, USA). TPGS, Kollisolv MCT70, Poloxamer 188, Tween 20, and Tween 80 were obtained as gifts from BASF (Ludwigshafen, Germany). Miglyol 812, Miglyol 829, Imwitor 491, Imwitor 900K, and Imwitor 960 were obtained as gifts from Cremer (Eatontown, NJ, USA). Labrafac, Compritol 888, Labrasol, and Gelucire 44/14 were obtained as gifts from Gattefossé (Saint Priest Cedex, France). Microcon Y-100 with molecular cutoff $100 \mathrm{kDa}$ was purchased from Millipore (Bedford, MA, USA). Ethanol USP grade was purchased from Pharmco-AAPER (Brookfield, CT, USA). Phosphoric acid was purchased from Sigma-Aldrich (St. Louis, MO, USA). Acetonitrile and methanol (HPLC grade) were purchased from Fisher Scientific (Pittsburgh, PA, USA). The sensitive prostate cancer cell lines, PC3 and DU145, were purchased from 
ATCC. PC3 and DU145 taxane-sensitive and -resistant cells were previously described [20]. Cells were maintained in Roswell Park Memorial Institute (RPMI) 1640 supplemented with 10\% fetal bovine serum (FBS) and 1\% penicillin-streptomycin. Cells were cultured at $37^{\circ} \mathrm{C}$ in a humidified incubator with $5 \% \mathrm{CO}_{2}$ and maintained in exponential growth phase by periodic subcultivation. Resistant cell lines were maintained with the addition of $5 \mathrm{nM}$ docetaxel in the culture medium. Identification of the cells were confirmed using short tandem repeat analysis.

\subsection{HPLC Analysis for Curcumin}

The concentration of CUR was quantified by high-performance liquid chromatography (HPLC) using a Waters Alliance HPLC system and an Inertsil ODS-3 column $(4.6 \mathrm{~mm} \times 150 \mathrm{~mm}, 5 \mu \mathrm{m}$ particle size, GL Sciences Inc., Torrance, CA, USA). The chromatography conditions were as follows: the mobile phase consisted of $0.1 \%$ orthophosphoric acid and acetonitrile $(40: 60, v / v)$ at a flow rate of $1.0 \mathrm{~mL} / \mathrm{min}$, and the effluent was detected at $420 \mathrm{~nm}$. The injection volume was $30 \mu \mathrm{L}$, and the analysis time was $6.5 \mathrm{~min}$ per sample. The retention time of CUR was $5.7 \mathrm{~min}$. The HPLC method was validated for the linear range, limitation of detection, accuracy, and precision. For the CUR standard curve, CUR was dissolved in methanol. The curve was found to be linear in the concentration range $0.1-50 \mu \mathrm{g} / \mathrm{mL}$ and $\mathrm{r}^{2}=0.998$.

\subsection{Screening of Oils and Surfactants}

The solubility of CUR was estimated in oils (Miglyol 812, Miglyol 829, Imwitor 491, Kollisolv MCT70, Labrafac, Imwitor 900K, Imwitor 960, and Compritol 888) and surfactants (Tween 20, Tween 80, Labrasol, Poloxamer 188, TPGS, and Gelucire 44/14). Oils and surfactants were accurately weighed into glass vials. An excess amount of CUR was added into glass vials containing an excipient. At each addition, the vials were stirred on a hot plate maintained at $65{ }^{\circ} \mathrm{C}$ for $3 \mathrm{~min}$. The drug solubility (drug/excipient, $w / w$ ) was measured.

\subsection{Preparation of Curcumin Nanoparticles}

NPs were prepared by an emulsion method as reported elsewhere with some modification [20]. Miglyol 812 (4 mg) and TPGS (4 mg) were heated to $35^{\circ} \mathrm{C}$. Two milliliters of deionized (D.I.) water pre-heated at $65{ }^{\circ} \mathrm{C}$ was added into the mixture of melted oils and surfactants. The mixture was stirred for $20 \mathrm{~min}$ and then cooled down to room temperature. To prepare CUR MT NPs, $75 \mu \mathrm{L}$ of $4 \mathrm{mg} / \mathrm{mL}$ of CUR dissolved in ethanol was directly added to the melted oil and surfactant; ethanol was removed by $\mathrm{N}_{2}$ stream and the NPs were prepared as described above.

\subsection{Solubility of Curcumin in Water and the Cell Medium}

The solubility of CUR was tested in D.I. water and the cell medium at $25^{\circ} \mathrm{C}$ for $48 \mathrm{~h}$, respectively. Briefly, excess CUR powders were added in each liquid. After $48 \mathrm{~h}$, the sample was centrifuged at 14,000 rpm for $5 \mathrm{~min}$. Then, the supernatant was transferred to an empty Eppendorf tube and centrifuged again at 14,000 $\mathrm{rpm}$ for $5 \mathrm{~min}$. The same procedure was repeated one more time. The concentration of CUR in the final supernatant was determined using HPLC assay.

\subsection{Particle Size and Zeta Potential Measurement}

Particle size and size distribution of NPs were measured using a Delsa Nano C Particle Size Analyzer (Beckman Coulter Inc., Brea, CA, USA). Ten microliters of NPs were diluted with $1 \mathrm{~mL}$ of D.I. water to reach the density range required by the instrument, and particle size analysis was performed at $90^{\circ}$ light scattering at $25^{\circ} \mathrm{C}$. PBS buffer $(10 \mu \mathrm{L})$ was added for the measurement of zeta potential by using the Analyzer. 


\subsection{Morphology of CUR MT NPS}

Five microliters of CUR MT NPs was applied to a carbon formvar-coated copper grid and stained with $2 \%$ aqueous uranyl acetate for $1 \mathrm{~min}$. Grids were visualized with a Tecnai $\mathrm{G}^{2}$ spirit transmission electron microscopy (TEM) (ThermoFisher, Hillsboro, OR, USA) equipped with a $\mathrm{LaB}_{6}$ source at $120 \mathrm{kV}$ using a Gatan Ultrascan CCD camera.

\subsection{Determination of Drug Loading and Entrapment Efficiency}

To quantify CUR in NPs, 1 part of CUR MT NPs was dissolved in 8 parts of methanol to measure DL\%. The EE\% was determined by separating free CUR and CUR MT NPs using Microcon Y-100 by centrifugation at $14,000 \mathrm{rpm}$ at $4{ }^{\circ} \mathrm{C}$, and then measuring CUR in the NPs. The DL $\%$ and EE\% were calculated based on the following equations:

$$
\begin{gathered}
\mathrm{EE} \%=\frac{\text { drug entrapped in NPs }}{\text { total drug added into NP preparation }} \times 100 \%(w \mid w) \\
\mathrm{DL} \%=\frac{\text { drug added }}{\text { weight of oil }} \times 100 \%(w \mid w)
\end{gathered}
$$

\subsection{Particle Size Stability of Nanoparticles at $4{ }^{\circ} \mathrm{C}$ and $37^{\circ} \mathrm{C}$}

The physical stability of CUR MT NPs was evaluated during their storage at $4{ }^{\circ} \mathrm{C}$ for 6 months. The stability of blank MT NPs and CUR MT NPs was also analyzed at $37^{\circ} \mathrm{C}$ in $10 \mathrm{mM} \mathrm{PBS}$ (pH 7.4) by adding $100 \mu \mathrm{L}$ NPs to $13 \mathrm{~mL}$ PBS with a shaker at $150 \mathrm{rpm}$. At each time interval, an aliquot was removed and allowed to equilibrate to room temperature before measuring particle size.

\subsection{Differential Scanning Calorimetry}

The analysis was performed to determine the physical state of CUR using a DSC (Perkin Elmer 4000, Waltham, MA, USA). Blank MT NPs and CUR MT NPs were concentrated about 50-fold using Microcon Y-100 at $4{ }^{\circ} \mathrm{C}$. The concentrated NPs were transferred to an aluminum pan and placed at room temperature for $48 \mathrm{~h}$. The heating curves were recorded at a scan rate of $10^{\circ} \mathrm{C} / \mathrm{min}$ from $20^{\circ} \mathrm{C}$ to $250^{\circ} \mathrm{C}$.

\subsection{In Vitro Release Study}

CUR release studies $(n=3)$ were completed at $37^{\circ} \mathrm{C}$ using PBS as the release medium. CUR MT NPs $(200 \mu \mathrm{L})$ was added into $20 \mathrm{~mL}$ phosphate buffered saline (PBS) and shaken at $135 \mathrm{rpm}$ at $37^{\circ} \mathrm{C}$. At each time interval, $500 \mu \mathrm{L}$ of sample was withdrawn. Released CUR was separated from CUR MT NPs using Microcon Y-100, and measured by HPLC as described above.

\subsection{Lyophilization of CUR MT NPS}

To determine the effect of lyophilization on the NPs, blank and CUR MT NPs were lyophilized using a freeze drier (VirTis Wizard 2.0, SP Industries, Gardiner, NY, USA). One milliliter of each sample was quickly frozen at $-40{ }^{\circ} \mathrm{C}$ and then lyophilized using a program of $1 \mathrm{~h}$ at $-10{ }^{\circ} \mathrm{C}$ for primary drying and $7 \mathrm{~h}$ at $15^{\circ} \mathrm{C}$ for secondary drying at 200 mTorr. The resultant lyophilized product was reconstituted in $1 \mathrm{~mL}$ of D.I. water and shaken until the sample became homogeneous. The particle size, P.I., zeta potential, EE\%, and DL\% were measured as described above.

\subsection{Cytotoxicity Studies}

The cytotoxicity was tested in sensitive and resistant DU145 and PC3 cells using 3-(4,5dimethylthiazol-2-yl)-2,5-diphenyltetrazolium bromide (MTT) assay. Cells were seeded into 96-well plates at 7000 cells/well and incubated at $37^{\circ} \mathrm{C}$ for $24 \mathrm{~h}$ under $5 \% \mathrm{CO}_{2}$. After overnight incubation, 
cells were treated with CUR, CUR MT NPs, and blank MT NPs at a concentration range from 0-270 $\mu \mathrm{M}$. Due to low water solubility, we dissolved CUR in DMSO and controlled DMSO less than 3\% in the treatment solutions. After $72 \mathrm{~h}$ of incubation, the medium was removed from each well and replaced with $90 \mu \mathrm{L}$ of DMEM medium without phenol red and MTT (10 $\mu \mathrm{L}$ of $5 \mathrm{mg} / \mathrm{mL}$ in PBS). After incubating for $4 \mathrm{~h}$ at $37{ }^{\circ} \mathrm{C}$ with $5 \% \mathrm{CO}_{2}, 0.04 \mathrm{~N} \mathrm{HCl}$ /isopropanol was added to each well and incubated for $5 \mathrm{~min}$ to dissolve formazan crystals formed by viable cells. The absorbance was measured at a test wavelength of $570 \mathrm{~nm}$ and a reference wavelength of $650 \mathrm{~nm}$ using the Synergy H1 Hybrid Multi-Mode Reader (BioTek, Winooski, VT, USA).

\subsection{Statistical Analysis}

Statistical comparisons were made with ANOVA, followed by pair-wise comparisons using Student's $t$ test using GraphPad Prism 6.0 (GraphPad Software, Inc., San Diego, CA). Results were considered significant at 95\% confidence interval $(p<0.05)$.

\section{Results}

\subsection{Selection of Oils and Surfactants and Preparation of CUR MT NPS}

As shown in Table 1, CUR was found to have the highest solubility in Imwitor 900K among the oils. However, liquid oils could provide advantages over solid oils in terms of drug loading and NP stability [21]. The solubility of CUR in the tested surfactants was comparable. Hence, the results confirmed that the MT NP, composed of Miglyol 812 and TPGS, was optimal for CUR.

Table 1. Solubility of curcumin in oils and surfactants.

\begin{tabular}{cccc}
\hline Oil & Solubility $(w / w)$ & Surfactant & Solubility $(w / w)$ \\
\hline Miglyol 812 & 0.0153 & Tween 20 & 0.166 \\
Imwitor 491 & 0.0261 & Tween 80 & 0.141 \\
Kollisolv MCT 70 & 0.0179 & Labrasol & 0.173 \\
Labrafac & 0.0120 & Poloxamer 188 & 0.153 \\
Imwitor 900K & 0.0512 & TPGS & 0.164 \\
Imwitor 960 & 0.0488 & Gelucire 44/14 & 0.153 \\
Compritol 888 & 0.0160 & & \\
\hline
\end{tabular}

Note: $w / w=$ weight by weight (gram/gram).

\subsection{Solubility}

The solubility of CUR in water and in the cell medium was $0.203 \pm 0.053 \mu \mathrm{g} / \mathrm{mL}$ and $0.623 \pm 0.031 \mu \mathrm{g} / \mathrm{mL}$, respectively $(n=3)$.

\subsection{Particle Size and Entrapment Efficiency}

As shown in Table 2 and Figure 1, particle size of CUR MT NPs was 138.9 nm with narrow size distribution (P.I. < 0.3), and zeta potential was $-24.4 \mathrm{mV}$. The EE\% and DL\% of CUR MT NPs were $96.3 \pm 6 \%(n=3)$ and $3.6 \%$, respectively. A TEM image confirmed the formation of spherical CUR MT NPs with particle size less than $100 \mathrm{~nm}$.

Table 2. Physico-chemical properties of CUR MT NPs $(n=3)$.

\begin{tabular}{|c|c|c|c|c|c|c|}
\hline Formulations & CUR $(\mu \mathrm{g} / \mathrm{mL})$ & Particle size $^{a}(\mathrm{~nm}) *$ & P.I. * & Zeta Potential $(\mathrm{mV}) *$ & $\mathrm{EE} \%$ * & DL\%* * \\
\hline Blank MT NPs & $\mathrm{n} / \mathrm{a}$ & $119.9 \pm 3.5$ & $0.246 \pm 0.016$ & $-20.6 \pm 1.9$ & $\mathrm{n} / \mathrm{a}$ & $\mathrm{n} / \mathrm{a}$ \\
\hline
\end{tabular}

a The data are presented as the mean particle size of NPs in different batches $\pm \mathrm{SD}(n=3) .{ }^{*} p>0.05$ when CUR MT NPs and lyophilized CUR MT NPs (Lyo CUR MT NPs) for each labeled parameter are compared. 


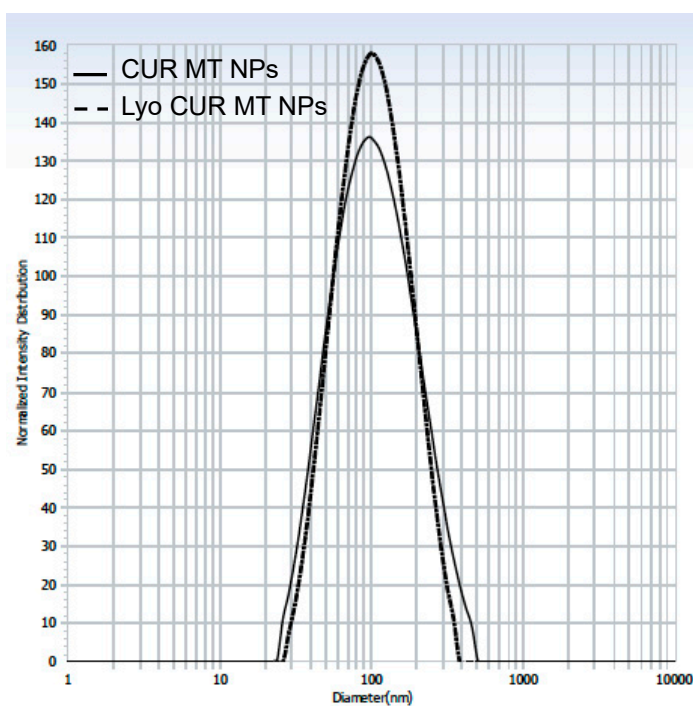

(A)

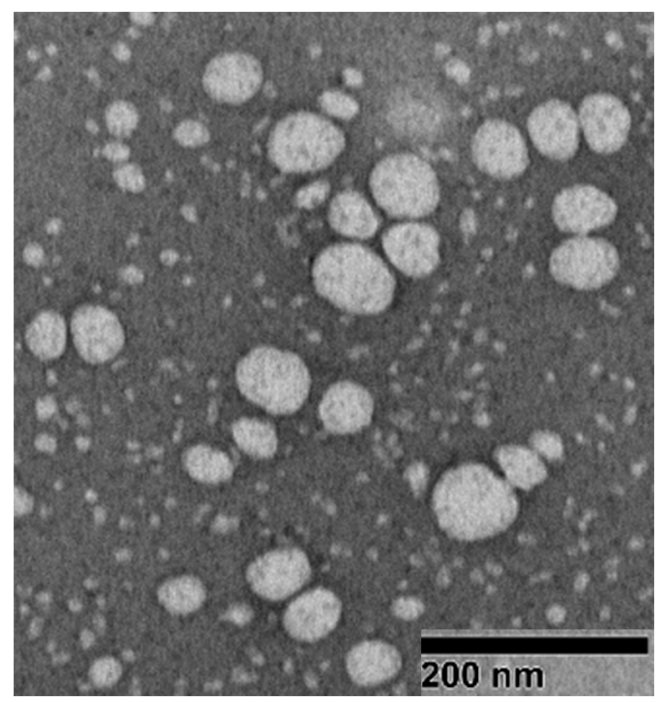

(B)

Figure 1. Particle size and size distribution of CUR MT NPs and Lyo CUR MT NPs. Particle size was measured by dynamic light scattering (CUR MT NPs and Lyo CUR MT NPs) (A) and TEM (CUR MT NPs) (B). Lyo CUR MT NPs were obtained by reconstitution of lyophilized CUR MT NPs.

\subsection{Physical Stability}

The physical stability of blank MT NPs and CUR MT NPs was monitored at $4{ }^{\circ} \mathrm{C}$ over 6 months for long-term storage and at $37^{\circ} \mathrm{C}$ in the PBS buffer for short-term stability in a simulated physiological condition. Both blank MT NPs and CUR MT NPs showed no significant changes in particle size at $4{ }^{\circ} \mathrm{C}$ over 6 months (Figure 2). Also, there were no significant changes in particle size for both blank MT NPs and CUR MT NPs incubated at $37^{\circ} \mathrm{C}$ in PBS over $96 \mathrm{~h}$ (Figure 3).

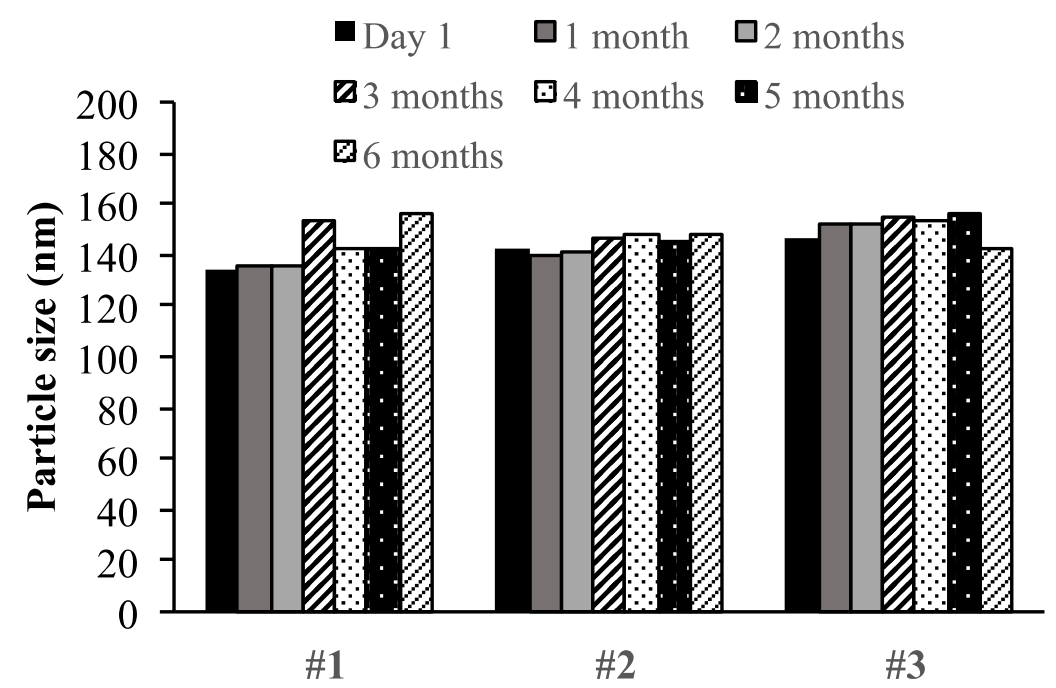

Figure 2. Long-term stability of CUR MT NPs stored at $4{ }^{\circ} \mathrm{C}$. Three different batches of CUR MT NPs were monitored for particle size over 6 months. For all tested samples, P.I. $<0.25$. Data are presented as the mean particle size of each batch. Particle size did not significantly change over the tested period ( $p>0.05$ for each batch). 


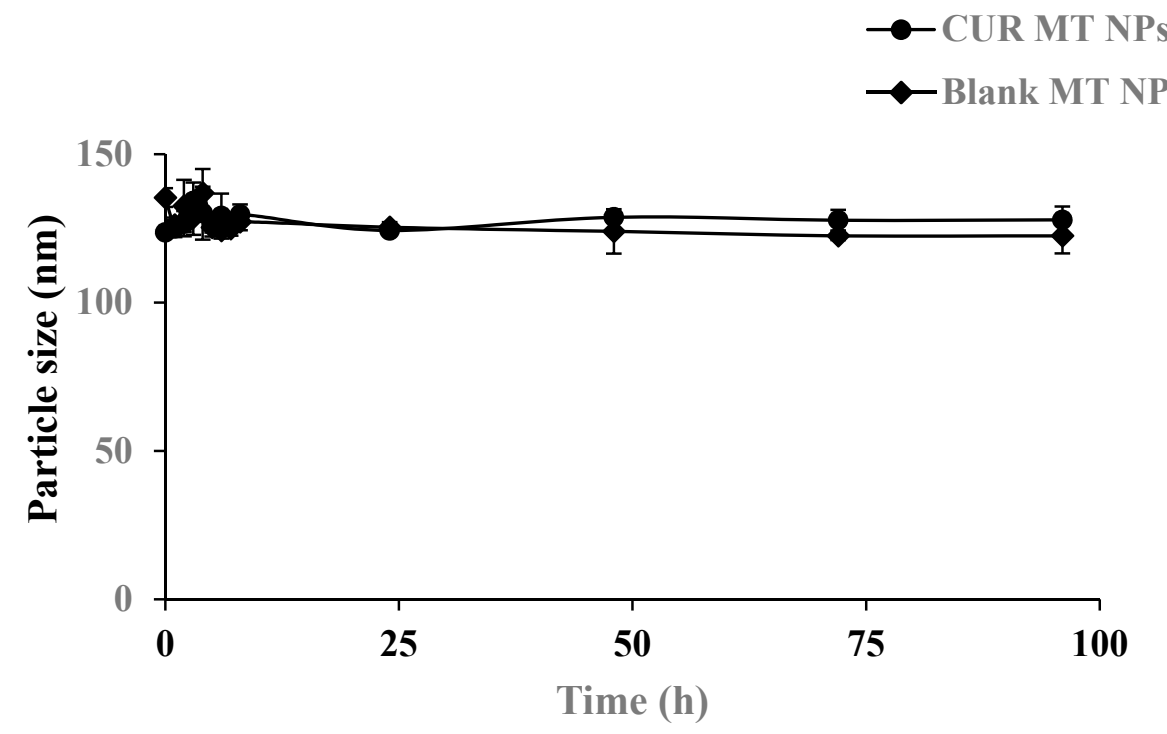

Figure 3. Physical stability of CUR MT NPs in a simulated physiological condition. The study was performed in PBS ( $\mathrm{pH} 7.4$ ) at $37^{\circ} \mathrm{C}$ for up to $96 \mathrm{~h}$. Blank MT NPs were used as a comparison. After equilibrating to room temperature, particle size was measured by dynamic light scattering. For all tested samples, P.I. $<0.25$. Data are presented as the mean $\pm \mathrm{SD}(n=3)$. There was no significant difference in particle size over $96 \mathrm{~h}$ within the group or between the groups $(p>0.05)$.

\subsection{Differential Scanning Calorimetry Analysis}

The melting peak of pure CUR was observed at $186^{\circ} \mathrm{C}$ (Figure 4). Compared to pure CUR, there was no peak around $186^{\circ} \mathrm{C}$ for CUR MT NPS, indicating that CUR was present as amorphous in the NPs. The melting endothermic peaks at $38^{\circ} \mathrm{C}$ in both blank MT NPs and CUR MT NPs were the melting peak of TPGS.

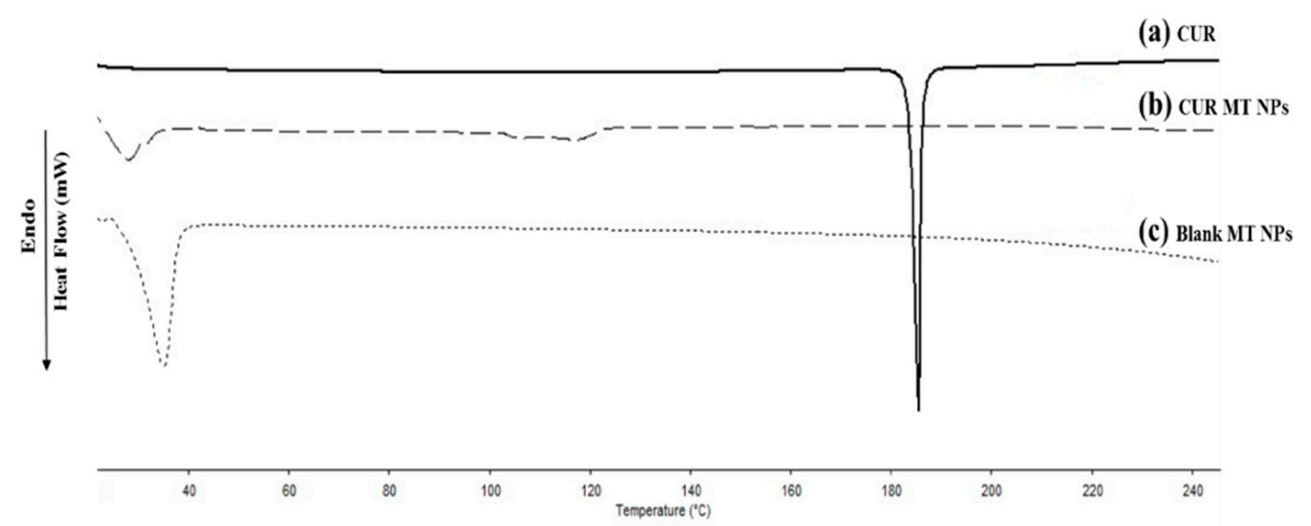

Figure 4. Differential scanning calorimetry (DSC) analysis of (a) CUR, (b) CUR MT NPs, and (c) blank MT NPs.

\subsection{In Vitro Release of Curcumin from the Nanoparticles}

The in vitro release profile of CUR MT NPs was tested in PBS at $37^{\circ} \mathrm{C}$. The cumulative CUR release from CUR MT NPs is shown in Figure 5. CUR showed a burst release from CUR MT NPs at the beginning and then continually released over $5 \mathrm{~h}$. 


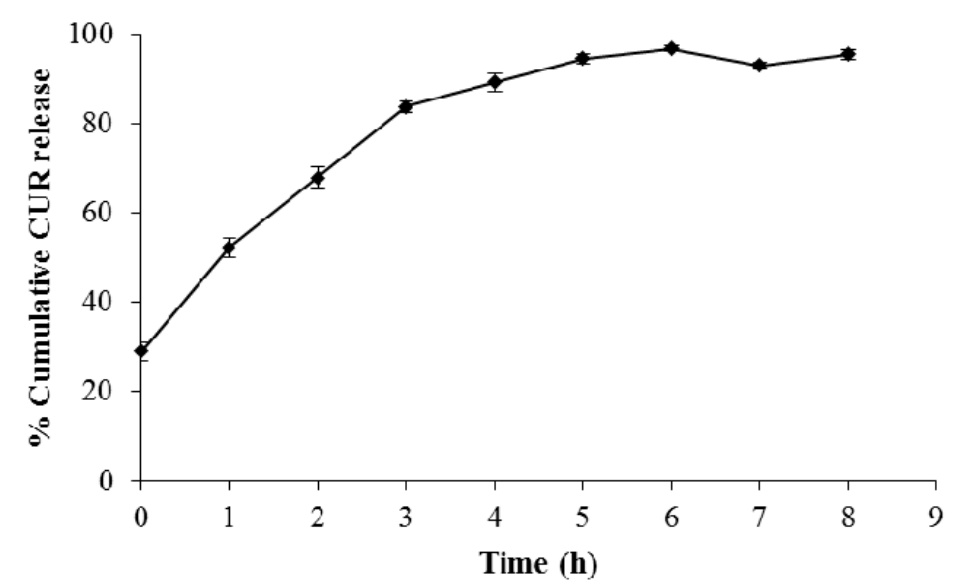

Figure 5. Release of CUR from CUR MT NPs in a simulated physiological condition. The study was performed in PBS (pH 7.4) at $37^{\circ} \mathrm{C}$ for $8 \mathrm{~h}$. Released CUR was separated from CUR MT NPs by Microcon-Y100 and measured by HPLC. Data are presented as the mean $\pm \operatorname{SD}(n=3)$.

\subsection{Lyophilization}

The rehydrated lyophilized CUR MT NPs (Lyo CUR MT NPs) had 93.1\% of EE\% and $3.5 \%$ of DL\%. As shown in Figure 1, CUR MT NPs and Lyo CUR MT NPs had similar particle size and size distribution. Based on statistical analysis, lyophilization did not change the physio-chemical properties of CUR MT NPs, including particle size, P.I., zeta potential, EE\%, and DL $\%(p>0.05)$ (Table 2 and Figure 1).

\subsection{In Vitro Cytotoxicity Studies}

Figure 6 shows the results of cytotoxicity studies of CUR and CUR MT NPs in sensitive and resistant PC 3 cells and sensitive and resistant DU145 cells, and Figure 7 shows the statistical comparison of the $\mathrm{IC}_{50} \mathrm{~s}$ of these treatments. As shown in Figure 7A, free CUR showed the same cytotoxicity in sensitive and resistant of PC 3 cell lines $(p>0.05)$. In contrast, the $\mathrm{IC}_{50}$ value of free CUR was 1.4-fold higher in resistant DU145 than that in sensitive DU145 (Figure 7B). Compared to free CUR, CUR MT $\mathrm{NP}$ decreased the $\mathrm{IC}_{50}$ value over 5 -fold in both sensitive and resistant PC 3 cell lines and about 2-fold in both sensitive and resistant DU145 cell lines (Figure 7A,B). Importantly, CUR MT NPs showed no significant differences in the $\mathrm{IC}_{50}$ values in sensitive and their matching resistant cells for both $\mathrm{PC} 3$ and DU145 (Figure 7A,B) $(p>0.05)$. The $\mathrm{IC}_{50}$ s of blank MT NPs were higher than $270 \mu \mathrm{M}$ in the tested cells, indicating low cytotoxicity of blank MT NPs. 


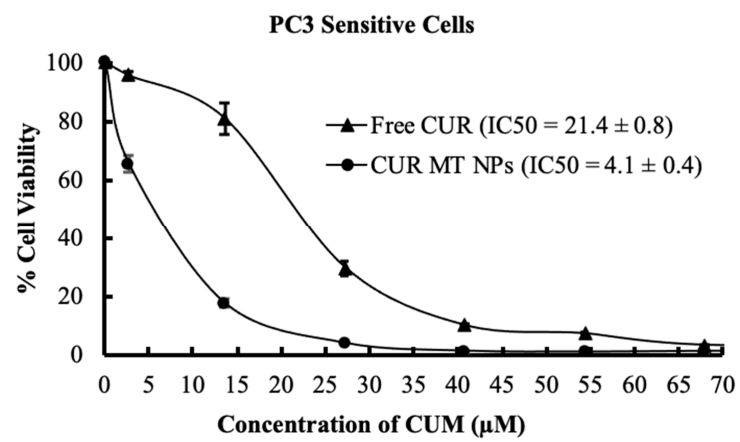

(A)

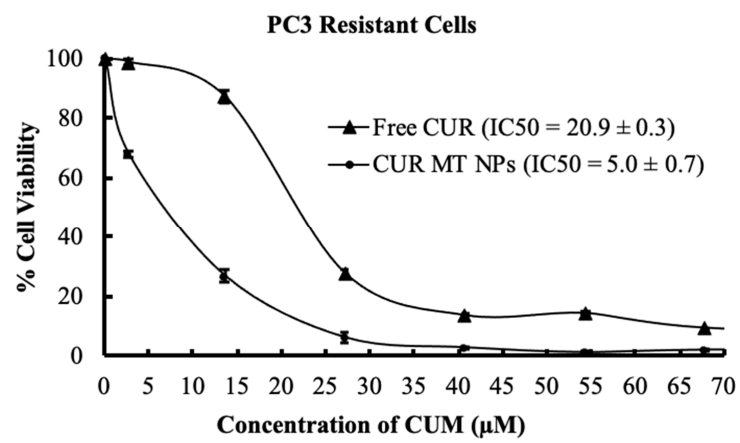

(B)

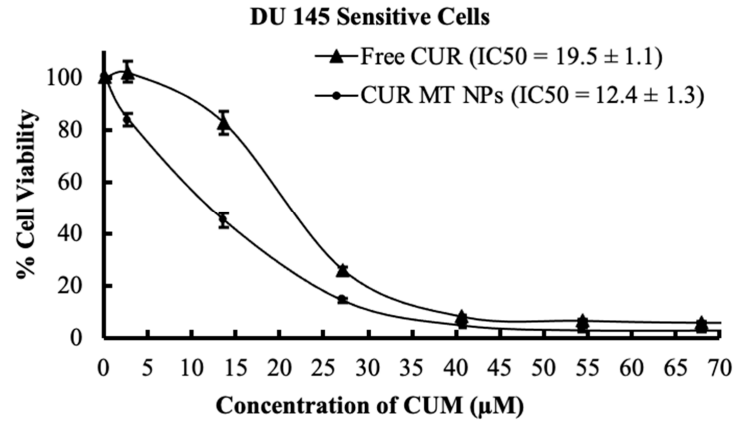

(C)

DU 145 Resistant Cells

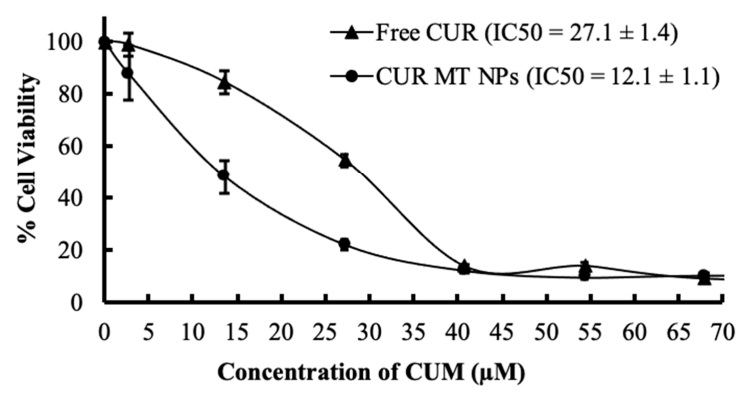

Figure 6. Cytotoxicity studies of CUR and CUR MT NPs in castration-resistant prostate cancer (CRPC) cells. (A) DTX-sensitive PC3 cells, (B) DTX-resistant PC3 cells, (C) DTX-sensitive DU145 cells, and (D) DTX-resistant DU145 cells. Data are presented at the mean of three independent measurements $(n=3) \pm \mathrm{SD}$. Cells were treated for $72 \mathrm{~h}$ with samples. Cell viability was measured by 3-(4,5-dimethylthiazol-2-yl)-2,5-diphenyltetrazolium bromide (MTT) assay. 


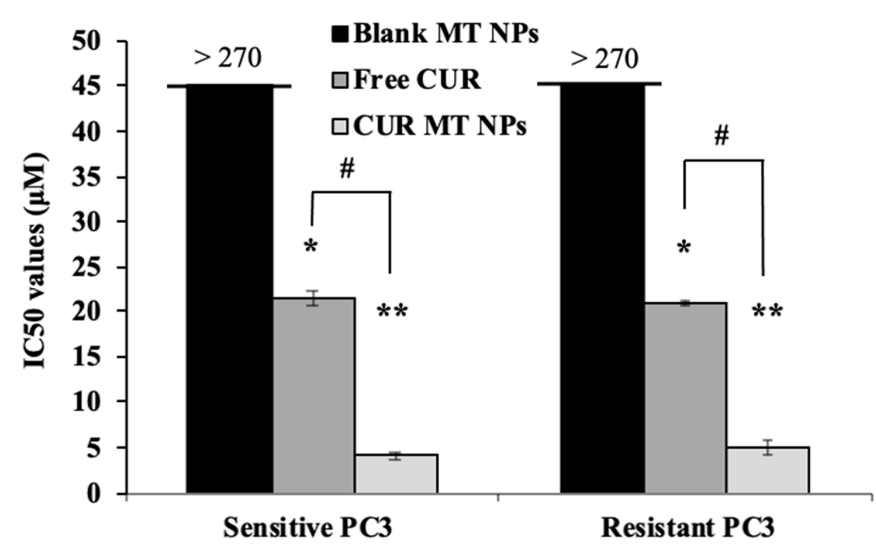

(A)

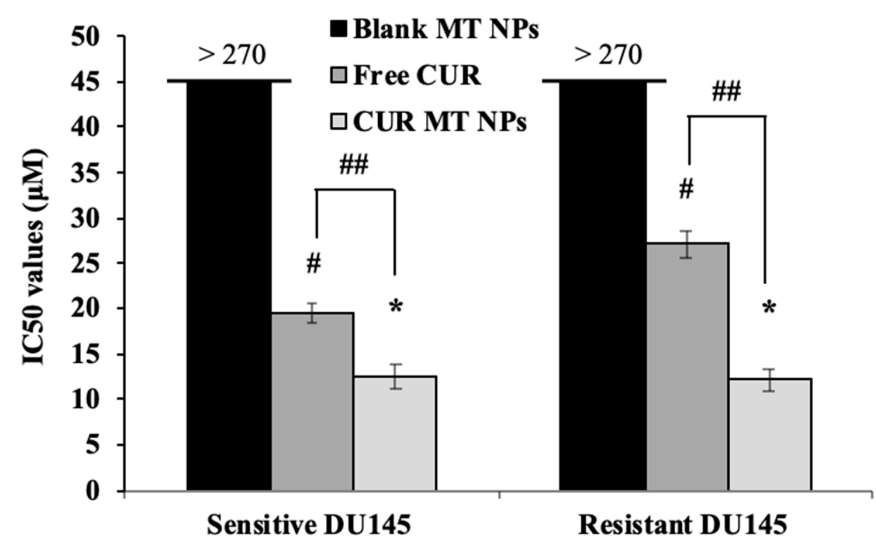

(B)

Figure 7. Comparison of $\mathrm{IC}_{50}$ s for CUR, CUR MT NPs, and blank MT NPs in CRPC cells. (A) $\mathrm{IC}_{50}$ values $(\mu \mathrm{M})$ of free CUR and CUR MT NPS in DTX-sensitive and -resistant PC3 cells. (B) IC 50 values $(\mu \mathrm{M})$ of free CUR and CUR MT NPS in DTX-sensitive and -resistant DU145 cells. Data are presented at the mean of three independent measurements $(n=3) \pm$ SD. \#, \#\# $p<0.05$ within the compared treatments; ${ }^{*}{ }^{* *} p>0.05$ within the compared treatments.

\section{Discussion}

CUR has been studied in many clinical trials for various cancers with little success although CUR exhibits strong activity for cancer prevention in animals. It is well known that CUR has low solubility; however, no study to date has reported water solubility data for CUR. According to our measurement, solubility of CUR in water is only $0.202 \mu \mathrm{g} / \mathrm{mL}$. Moreover, solubility of CUR increased over 3-fold in the cell medium compared to water, demonstrating that the composition of the solution has great influence on CUR solubility. It is possible that the variation of CUR solubility in different environments could cause controversial results from lab studies and human studies. On the other hand, the poor solubility confirmed that CUR must be formulated in such a way as to overcome the solubility issues in order to apply its therapeutic effect.

We developed CUR MT NPs in this study. Our CUR NPs demonstrated stability at $4{ }^{\circ} \mathrm{C}$ over 6 months (Figure 2) and at $37^{\circ} \mathrm{C}$ over $96 \mathrm{~h}$ (Figure 3). Moreover, CUR MT NPs are lyophilizable without losing their properties, which grants the other opportunity for long-term storage (Table 2 and Figure 1 ). Liquid lipid Miglyol 812 reduces particle crystallinity, conferring better stability and suitability than solid lipids [22]. The optimal 6-month stability of CUR MT NPs could result from the use of liquid Miglyol 812 in the NPs. CUR MT NPs were smaller than $140 \mathrm{~nm}$ with narrow size distribution (Figure 1). Because NPs tend to aggregate during long-term storage, lyophilization is a promising 
method to remove water and keep NPs in dry states for long-term storage. However, lyophilization often causes an increase of particle size even with cryoprotectants. Interestingly, CUR MT NPs can be lyophilized without using cryoprotectants. After reconstitution, properties of lyophilized CUR MT NPs, including particle size, size distribution, DL and EE, did not change, which shows the potential of developing CUR MT NPs as a medical injection. Studies have suggested that particles between 100 to $200 \mathrm{~nm}$ are desired for adhesion to and interaction with cells as well as taking advantage of the EPR effect [23]. Thus, our particle size is desirable for cancer drug delivery. According to the DSC study, CUR dissolved in Miglyol 812 and TPGS during the NP preparation and existed as amorphous in the NPs (Figure 4). CUR MT NPs showed a relatively rapid, but complete release over $5 \mathrm{~h}$ (Figure 5), demonstrating that NPs enhanced the solubility of CUR and could facilitate the solubilization of CUR in the blood.

CUR has pleiotropic properties that modulate numerous targets including proteins (i.e., tubulin), transcription factors, growth factors and their receptors, cytokines, enzymes, and apoptosis [24,25]. Thus, CUR has been used for cancer therapy and prevention, and also as a chemosensitizer to combine with other drugs (e.g., DTX) to overcome drug resistance in various cancers [26]. However, there have been no studies of CUR itself as a therapeutic drug in DTX-resistant CRPC. Compelling evidence showed that NPs could overcome P-gp-mediated drug resistance in cancers [27-29]. In our previous studies, we demonstrated that the blank MT NPs inhibited P-gp function [20]. Thus, by combining multi-targeted behaviors and a wide spectrum of actions of CUR with the advantages of the MT NPs, we hypothesized that CUR MT NPs could be potentially used as a therapeutic drug to overcome DTX-resistant CRPC. It is known that the resistance in DU145 and PC3 cells is controlled by different resistant mechanisms [7]. Overexpression of P-gp has been identified as the only main cause of resistance in DU145 cells, but P-gp does not play a role in resistant PC3 cells [8]. Alterations of microtubule formation, down-regulation of microtubule-related genes, alterations in the expression of tubulin composition, and up-regulation of apoptosis genes were identified in the resistant PC3 cells [7]. To test our hypothesis, we studied the cytotoxicity of CUR MT NPs in both resistant and sensitive DU145 and PC3 cell lines.

Our data showed that free CUR had the same $\mathrm{IC}_{50} \mathrm{~s}$ in both sensitive and resistant PC 3 cells, indicating that. in spite of resistance to DTX, PC3-resistant cells were not resistant to free CUR (Figure 7A). In contrast, the $\mathrm{IC}_{50}$ of free CUR in DU145-resistant cells was higher than that in DU145-sensitive cells, indicating, in addition to resistance to DTX, DU145-resistant cells are also resistant to CUR (Figure 7B). According to the results, CUR MT NPs were 5-fold more potent than free CUR in PC3 cells lines and about 2-fold more potent than free CUR in DU145 cells, demonstrating that the NPs enhanced CUR cytotoxicity in these cancer cells (both sensitive and resistant cells). Importantly, the $\mathrm{IC}_{50}$ s of CUR MT NPs did not have significant differences between sensitive and resistant PC 3 cells $(4.1 \pm 0.4 \mu \mathrm{M}$ vs. $5.0 \pm 0.7 \mu \mathrm{M}, p>0.05)$ or between sensitive and resistant DU145 cells $(12.4 \pm 1.3 \mu \mathrm{M}$ vs. $12.1 \pm 1.1 \mu \mathrm{M}, p>0.05)$ (Figure 7A,B), which demonstrated that CUR MT NPs completely overcame the resistance not only in PC3 cells but also in DU145 cells. The results demonstrate that the combination of CUR (a multi-targeted agent) and MT NP (a drug-carrier inhibiting P-gp) really synergize their advantages and provide a novel therapeutic strategy for the treatment of DTX-resistant CRPC. Considering that CUR is a relatively safe compound, CUR MT NPs with the $\mathrm{IC}_{50} \mathrm{~s}$ around $10 \mu \mathrm{M}$ will be an effective therapeutic drug for the treatment of DTX-resistant CRPC.

Further cell studies (e.g., cell uptake studies, apoptosis, and the state of autophagy) are expected to understand the underlying mechanisms, and animal studies are granted to demonstrate the therapeutic efficacy of CUR MT NPs. To the best of our knowledge, this is the first report to use CUR NPs for the treatment of DTX-resistant CRPC. This study also demonstrates the therapeutic potential of CUR for the treatment of other drug-resistant cancers. 


\section{Conclusions}

Taken together, we are the first to demonstrate that CUR MT NPs have potential to overcome DTX-resistant CRPC. We successfully developed and characterized CUR MT NP with high DL and high EE. CUR MT NPs were stable at $4{ }^{\circ} \mathrm{C}$ over 6 months. They could be lyophilized without using cryoprotectants. In addition to increasing the potency of CUR in sensitive cancer cells, CUR MT NPs completely overcame resistance and restored CUR potency in both resistant PC 3 and DU145 cells. By integrating the advantages of CUR and MT NPs, CUR MT NPs have potential as a novel therapeutic drug to overcome DTX resistance in CRPC.

Author Contributions: I.T., A.T., P.P., and J.T.N. performed the experiments. I.T., A.T., and X.D. designed the experiments and interpreted the data. I.T. and X.D. wrote the paper. E.T.K. edited the paper. X.D. supervised the project. All authors have read and agreed to the published version of the manuscript.

Funding: This research was supported by an intramural grant from the University of North Texas Health Science Center.

Conflicts of Interest: The authors declare no conflicts of interest.

\section{References}

1. Bray, F.; Ferlay, J.; Soerjomatraram, I.; Siegel, R.L.; Torr, L.A.; Jemal, A. Global cancer statistics 2018: GLOBOCAN estimates of incidence and mortality worldwide for 36 cancers in 185 countries. CA Cancer J. Clin. 2018, 68, 394-424. [CrossRef] [PubMed]

2. Karantanos, T.; Corn, P.G.; Thompson, T.C. Prostate cancer progression after androgen deprivation therapy; mechanisms of castrate resistance and novel therapeutic approaches. Oncogene 2013, 32, 5501-5511. [CrossRef] [PubMed]

3. Tannock, I.F.; De Wit, R.; Berry, W.R.; Horti, J.; Pluzanska, A.; Chi, K.N.; Oudard, S.; Theodore, C.; James, N.D.; Turesson, I.; et al. Docetaxel plus prednisone or mitoxantrone plus prednisone for advanced prostate cancer. N. Engl. J. Med. 2004, 351, 1502-1512. [CrossRef] [PubMed]

4. Brooks, T.; Minderman, H.; O’Loughlin, K.L.; Pera, P.; Ojima, I.; Baer, M.R.; Bernacki, R.J. Taxane-based reversal agents modulate drug resistance mediated by P-glycoprotein, multidrug resistance protein, and breast cancer resistance protein. Mol. Cancer Ther. 2003, 2, 1195-1205.

5. Goldman, B. Multidrug resistance: Can new drugs help chemotherapy score against cancer? J. Natl. Cancer Inst. 2003, 95, 255-257. [CrossRef]

6. Thomas, H.; Coley, H.M. Overcoming multidrug resistance in cancer: An update on the clinical strategy of inhibiting p-glycoprotein. Cancer Control. 2003, 10, 159-165. [CrossRef]

7. Takeda, M.; Mizokami, A.; Mamiya, K.; Li, Y.Q.; Zhang, J.; Keller, E.T.; Namiki, M. The establishment of two paclitaxel-resistant prostate cancer cell lines and the mechanisms of paclitaxel resistance with two cell lines. Prostate 2007, 67, 955-967. [CrossRef]

8. Tiwari, A.K.; Sodani, K.; Dai, C.L.; Ashby, C.R., Jr.; Chen, Z.S. Revisiting the ABCs of multidrug resistance in cancer chemotherapy. Curr. Pharm. Biotechnol. 2011, 12, 570-594. [CrossRef]

9. Aggarwal, B.B.; Shishodia, S. Molecular targets of dietary agents for prevention and therapy of cancer. Biochem. Pharmacol. 2006, 71, 1397-1421. [CrossRef]

10. Shishodia, S.; Chaturvedi, M.M.; Aggarwal, B.B. Role of curcumin in cancer therapy. Curr. Prob. Cancer 2007, 31, 243-305. [CrossRef]

11. Wilken, R.; Wang, V.M.; Srivatsan, E. Curcumin: A review of anti-cancer properties and therapeutic activity in head and neck squamous cell carcinoma. Mol. Cancer 2011, 10, 12. [CrossRef] [PubMed]

12. Zhao, H.; Yu, X.; Qi, R.; Shang, F.; Su, Z. Inhibitory Effects of curcumin in combination with paclitaxel on prostate cancer xenografted model. Progress Mod. Biomed. 2010, 10, 823-827.

13. Wang, Y.J.; Pan, M.H.; Cheng, A.L.; Lin, L.; Ho, Y.S.; Lin, J.K. Stability of curcumin in buffer solutions and characterization of its degradation products. J. Pharm. Biomed. Anal. 1997, 15, 1867-1876. [CrossRef]

14. Bar-Sela, G.; Epelbaum, R.; Schaffer, M. Curcumin as an anti-cancer agent: Review of the gap between basic and clinical applications. Curr. Med. Chem. 2010, 17, 190-197. [CrossRef]

15. Shoba, G.; Joy, D.; Joseph, T.; Majeed, M.; Rajendran, R.; Srinivas, P.S. Influence of piperine on the pharmacokinetics of curcumin in animals and human volunteers. Planta Med. 1998, 64, 353-356. [CrossRef] 
16. De Jong, W.H.; Borm, P.J. Drug delivery and nanoparticles: Applications and hazards. Int. J. Nanomed. 2013, 3, 133-149. [CrossRef]

17. Markman, J.L.; Rekechenetskiy, A.; Holler, E.; Ljubimova, J.Y. Nanomedicine therapeutic approaches to overcome cancer drug resistance. Adv. Drug Deliv. Rev. 2013, 65, 1866-1879. [CrossRef]

18. Grama, C.; Ankola, D.; Kumar, M. Poly (lactide-co-glycolide) nanoparticles for peroral delivery of bioactives. Curr. Opin. Colloid Interface Sci. 2011, 16, 238-245. [CrossRef]

19. Collnot, E.M.; Baldes, C.; Schaefer, U.F.; Edgar, K.J.; Wempe, M.F.; Lehr, C.M. Vitamin E-TPGS p-glycoprotein inhibition mechanism: Influence on conformational flexibility, intracellular ATP levels, and role of time and site of access. Mol. Pharmaceut. 2010, 7, 642-651. [CrossRef]

20. Tanaudommongkon, A.; Tanaudommongkon, I.; Prathipati, P.; Nguyen, J.T.; Dong, X. Development and Characterization of Docetaxel-Loaded Nanoparticles for Docetaxel-Resistant Castration-Resistant Prostate Cancer. J. Nanosci. Nanotech. 2017, 17, 3920-3926. [CrossRef]

21. Dong, X.; Mattingly, C.A.; Tseng, M.; Cho, M.; Adams, V.; Mumper, R.J. Development of new lipid-based paclitaxel nanoparticles using sequential simplex optimization. Eur. J. Pharm. Biopharm. 2008, 72, 9-17. [CrossRef] [PubMed]

22. Neves, A.R.; Lucio, M.; Martins, S.; Lima, J.L.; Reis, S. Novel resveratrol nanodelivery systems based on lipid nanoparticles to enhance its oral bioavailability. Int. J. Nanomed. 2013, 8, 177-187. [CrossRef]

23. Huang, L.; Liu, Y. In vivo delivery of RNAi with lipid-based nanoparticles. Annu. Rev. Biomed. Eng. 2011, 13, 507-530. [CrossRef] [PubMed]

24. Maheshwari, R.K.; Singh, A.K.; Gaddipati, J.; Srimal, R.C. Multiple biological activities of curcumin: A short review. Life Sci. 2006, 78, 2081-2087. [CrossRef] [PubMed]

25. Jurenka, J.S. Anti-inflammatory properties of curcumin, a major constituent of curcuma longa: A review of preclinical and clinical research. Altern. Med. Rev. 2009, 14, 141-153. [PubMed]

26. Banerjee, S.; Singh, S.K.; Chowdhury, I.; Lillard, J.W.; Singh, R. Combinatorial effect of curcumin with docetaxel modulates apoptotic and cell survival molecules in prostate cancer. Front. Biosci. 2017, 9, 235-245. [CrossRef]

27. Dong, X.; Mattingly, C.; Tseng, M.T.; Cho, M.J.; Liu, Y.; Adams, V.R.; Mumper, R.J. Doxorubicin and paclitaxel-loaded lipid-based nanoparticles overcome multidrug resistance by inhibiting p-glycoprotein and depleting ATP. Cancer Res. 2009, 69, 3918-3926. [CrossRef]

28. Xue, X.; Liang, X.-J. Overcoming drug efflux-based multidrug resistance in cancer with nanotechnology. Chi. J. Cancer 2012, 31, 100-109. [CrossRef]

29. Niazi, M.; Zakeri-Milani, P.; Najafi Hajivar, S.; Soleymani Goloujeh, M.; Ghobakhlou, N.; Shahbazi Mojarrad, J.; Valizadeh, H. Nano-based strategies to overcome p-glycoprotein-mediated drug resistance. Expert Opin. Drug Metab. Toxicol. 2016, 12, 1021-1033. [CrossRef] 